\title{
Emergent snake magnetic domains in canted kagome ice
}

\author{
Wen-Han Kao (高文瀚) $0,{ }^{1}$ Gia-Wei Chern, ${ }^{2}$ and Ying-Jer Kao (高英哲) $\oplus^{1,3,4, *}$ \\ ${ }^{1}$ Department of Physics and Center for Theoretical Physics, National Taiwan University, Taipei 10607, Taiwan \\ ${ }^{2}$ Department of Physics, University of Virginia, Charlottesville, Virginia 22904, USA \\ ${ }^{3}$ National Center for Theoretical Sciences, National Tsing Hua University, Hsinchu 30013, Taiwan \\ ${ }^{4}$ Department of Physics, Boston University, 590 Commonwealth Avenue, Boston, Massachusetts 02215, USA
}

(Received 28 August 2019; revised manuscript received 23 January 2020; accepted 19 February 2020; published 16 April 2020)

\begin{abstract}
We study the two-dimensional kagome-ice model derived from a pyrochlore lattice with second- and thirdneighbor interactions. The canted moments align along the local $\langle 111\rangle$ axes of the pyrochlore and respond to both in-plane and out-of-plane external fields. We find that the combination of further-neighbor interactions together with the external fields introduces a rich phase diagram with different spin textures. Close to the phase boundaries, metastable "snake" domains emerge with extremely long relaxation time. Our kinetic Monte Carlo analysis of the magnetic-field quench process from saturated state shows unusually slow dynamics. Although the interior spins are almost frozen in snake domains, the spins on the edge are free to fluctuate locally, leading to frequent creation and annihilation of monopole-antimonopole bound states. Once the domains are formed, these excitations are localized and can hardly propagate because of the energy barrier of snakes. The emergence of such snake domains may shed light on the experimental observation of dipolar spin ice under tilted fields and provide a new strategy to manipulate both spin and charge textures in artificial spin ice.
\end{abstract}

DOI: 10.1103/PhysRevResearch.2.023046

\section{INTRODUCTION}

Metastability in physical systems indicates a rough free energy landscape with multiple nearly degenerate local minima. Normally, it requires random disorder for the metastable behavior to occur, with spin glass as the most prominent example [1]. On the other hand, disorder-free geometrically frustrated spin-ice compounds, such as $\mathrm{Ho}_{2} \mathrm{Ti}_{2} \mathrm{O}_{7}$ and $\mathrm{Dy}_{2} \mathrm{Ti}_{2} \mathrm{O}_{7}$, also suffer from spin freezing at low temperature due to the slow dynamics originating from the extensive quasidegenerate lowenergy states and the topological constraint known as the ice rule [2,3]. For a pyrochlore lattice with corner-sharing tetrahedra, the ice rule dictates that the spins in each tetrahedron should obey the two-in-two-out constraint, resulting in a divergence-free flux field on the dual diamond lattice. The icerule constraint originating from the effective nearest-neighbor coupling of spins can be regarded as a conservation law of emergent gauge fields, giving rise to the anisotropic dipolar correlation [4]. The long-range dipolar interaction in the more sophisticated dipolar spin ice (DSI) model merely provides correction to the projectively equivalent band of low-energy states [5]; the quasidegenerate ice manifold remains as a stable low-temperature phase over a wide temperature window [6].

\footnotetext{
*yjkao@ phys.ntu.edu.tw

Published by the American Physical Society under the terms of the Creative Commons Attribution 4.0 International license. Further distribution of this work must maintain attribution to the author(s) and the published article's title, journal citation, and DOI.
}

Remarkably, excitations upon this ice-rule manifold are fractionalized quasiparticles with magnetic Coulomb interaction, leading to the resemblance of magnetic monopole $[7,8]$ and the emergence of an unusual spin liquid called the Coulomb phase $[9,10]$. Furthermore, the theoretical proposal of spin fragmentation shows that Coulomb phase can occur naturally from non-divergence-free systems and coexist with long-range ordered monopoles [11]. Experimental signatures of spin fragmentation can be found in various systems such as quantum spin ice $\mathrm{Nd}_{2} \mathrm{Zr}_{2} \mathrm{O}_{7}$ [12], tripod kagome compound $\mathrm{Dy}_{3} \mathrm{Mg}_{2} \mathrm{Sb}_{3} \mathrm{O}_{14}[13,14]$, and thermally active artificial kagome array of GdCo alloy [15]. The interplay between geometrical frustration and interacting topological charges brings about complex spin textures [16-19] and intriguing out-of-equilibrium phenomena [20-22].

Under a [111] magnetic field, the pyrochlore spin ice exhibits a two-stage magnetization process [23]. The first plateau refers to the kagome-ice phase with residual spin degeneracy, and the second plateau is a long-range ordered state which can be interpreted as a magnetic monopole condensate [7]. Neutron scattering experiments reveal the pinch point singularity of the kagome-ice phase by applying a magnetic field along [111] crystallographic direction on $\mathrm{Ho}_{2} \mathrm{Ti}_{2} \mathrm{O}_{7}$, and an unusual critical scattering appears when the field is slightly tilted away from this direction [24]. By using the dipolar spin-ice model with combination of fields, it is shown that the critical scattering is ascribable to the long-range ordered $\mathbf{q}=X$ state, and the lack of Bragg peak may be attributed to partial ordering in the real material [25].

In this paper, we study the interplay between furtherneighbor interactions and tilted magnetic fields in the kagome ice in order to understand possible mechanisms that prevent 
ordering. Specifically, we study here a two-dimensional classical kagome ice with magnetic moments aligned along the local $\langle 111\rangle$ axes originating from the pyrochlore lattice. Because of this canting, the moments can respond to both in-plane and out-of-plane fields, allowing us to explore the rich phase diagrams by tuning both the further-neighbor interactions and external fields. In particular, at the boundary between different phases, we find metastable winding snake domains emerge with local excitations of monopole-antimonopole pairs. Unlike in the conventional spin ice, these excitations are immobile and cannot proliferate to destabilize the snake domains. This leads to observable signatures in the neutron experiments and should provide a new perspective on the interpretation of experimental data.

The rest of the paper is organized as follows: In Sec. II, we introduce the model Hamiltonian and the geometry of canted moment and external fields. In Sec. III, we present the equilibrium Monte Carlo results and the phase diagrams of various ground states. To show the relaxation process from saturated state and the emergence of snake domains, we perform the rejection-free kinetic Monte Carlo simulation. The method and results are described in Sec. IV. Then, in Sec. V, we discuss the energetics of snake domain and the localized excitation on its periphery, along with possible experimental signatures in spin-ice crystals or artificial spin ices. We end with concluding remarks in the last section.

\section{THE MODEL}

We consider a spin model on the two-dimensional kagome lattice with Ising moments canted along the local easy axes derived from the pyrochlore ice (Fig. 1),

$$
\begin{aligned}
\mathcal{H}= & \bar{J}_{1} \sum_{\langle i j\rangle} \mathbf{S}_{i} \cdot \mathbf{S}_{j}+\bar{J}_{2} \sum_{\langle\langle i j\rangle\rangle} \mathbf{S}_{i} \cdot \mathbf{S}_{j}+\bar{J}_{3 a} \sum_{3 \mathrm{rd}(\mathrm{a})} \mathbf{S}_{i} \cdot \mathbf{S}_{j} \\
& +\bar{J}_{3 b} \sum_{3 \mathrm{rd}(\mathrm{b})} \mathbf{S}_{i} \cdot \mathbf{S}_{j}-\overline{\mathbf{h}} \cdot \sum_{i} \mathbf{S}_{i} .
\end{aligned}
$$

Each magnetic moment can be written as $\mathbf{S}_{i}=\mu \sigma_{i} \mathbf{d}_{\kappa(i)}$, where $\sigma_{i}= \pm 1$ is the Ising variable and $\mathbf{d}_{\kappa(i)}$ is the unit vector along local easy axis of the $i$ th spin belonging to sublattice $\kappa$. Writing the Hamiltoinian in terms of the Ising variables $\sigma_{i}$,

$$
\begin{aligned}
\mathcal{H}= & J_{1} \sum_{\langle i j\rangle} \sigma_{i} \sigma_{j}+J_{2} \sum_{\langle\langle i j\rangle\rangle} \sigma_{i} \sigma_{j}+J_{3 a} \sum_{3 \operatorname{rd}(\mathrm{a})} \sigma_{i} \sigma_{j} \\
& +J_{3 b} \sum_{3 \operatorname{rd}(\mathrm{b})} \sigma_{i} \sigma_{j}-\mathbf{h} \cdot \sum_{i} \mathbf{d}_{\kappa(i)} \sigma_{i} .
\end{aligned}
$$

In the following, we use the effective exchange interactions $J_{1}, J_{2}, J_{3 a}$, and $J_{3 b}$ to characterize the phases, and the external magnetic field $\mathbf{h}$ is decomposed into out-of-plane and in-plane components $\mathbf{h}=h_{\perp} \hat{\mathbf{n}}+h_{\|} \hat{\mathbf{k}}$ [26] as depicted in Fig. 1(a). Explicitly, the products of unit vectors and local easy axes read

$$
\hat{\mathbf{n}} \cdot \mathbf{d}_{\kappa}=-\frac{1}{3}\left(\begin{array}{l}
1 \\
1 \\
1
\end{array}\right), \hat{\mathbf{k}} \cdot \mathbf{d}_{\kappa}=-\frac{\sqrt{2}}{3}\left(\begin{array}{r}
1 \\
1 \\
-2
\end{array}\right) .
$$

In the following, we set $J_{1}=1$.

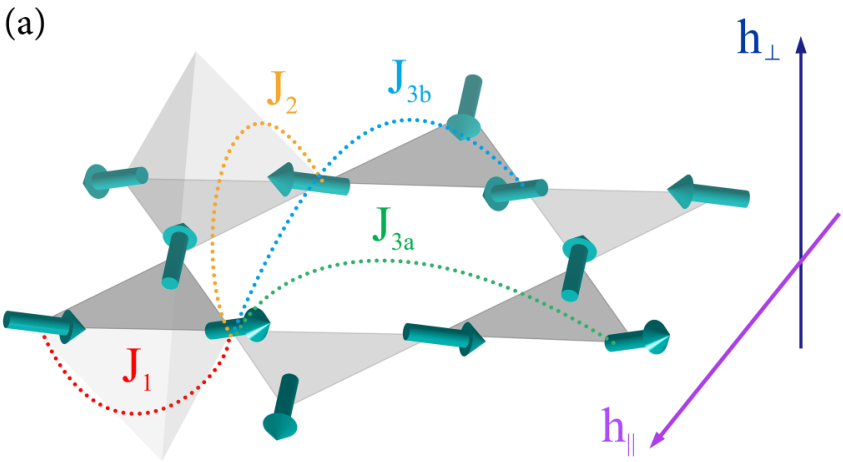

(b)

(c)
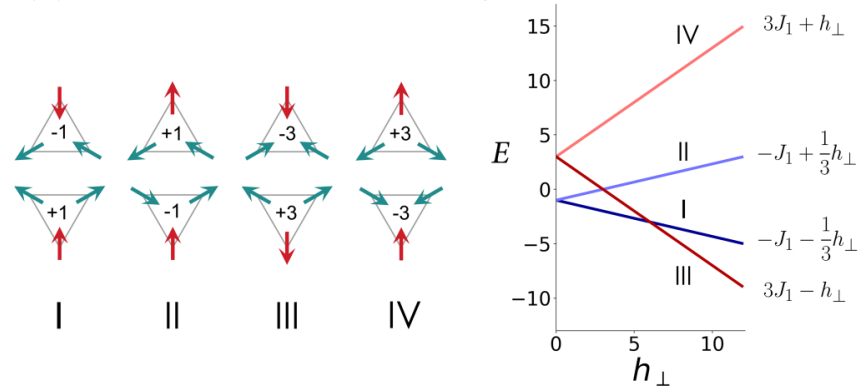

FIG. 1. Canted kagome ice model. (a) The easy direction of each moment (green arrows) is pointing toward the center of a virtual tetrahedron. (b) Triangular configurations with possible topological charges. The red vertical arrows represent kink spins, and the green horizontal arrows represent stripe spins. (c) Energy-level crossing of different charges as a function of the magnitude of perpendicular field. At higher fields, charges of group I and III are more favorable than II and IV.

\section{GROUND STATES}

With only the antiferromagnetic nearest-neighbor interaction $J_{1}>0$, the system has macroscopic degeneracy in the ground state. Each configuration in the ground-state manifold satisfies the ice rule on the kagome lattice: For both upwardpointing and downward-pointing triangles, the three spins must be either two-in-one-out or one-in-two-out. We can define the topological charge for the kagome ice as [27,28]

$$
Q_{\triangle}=\sum_{i \in \Delta} \sigma_{i}, \quad Q_{\nabla}=(-1) \sum_{i \in \nabla} \sigma_{i}
$$

for upward-pointing and downward-pointing triangles, respectively. The kagome-ice rule in this charge language becomes $Q_{\triangle}, Q_{\nabla}= \pm 1$ [Fig. 1(b)]. Therefore, the nearestneighbor Hamiltonian contains only self-energy of topological charges and describes a gas of single charges. The total charge density of the system is calculated through

$$
Q_{t}=\frac{1}{L^{2}}\left|\sum_{k} Q_{\triangle, k}\right|,
$$

such that a single- (triple-) charge ordered state gives $Q_{t}=$ $+1(+3)$. $L$ denotes the number of unit cells in each direction and the total number of spins $N=3 \times L \times L$. 
(a)

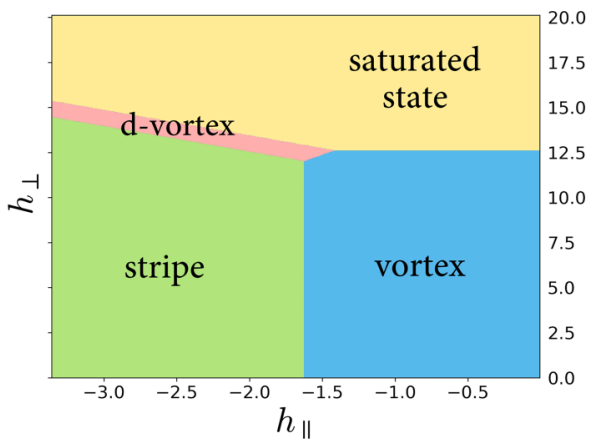

(b)

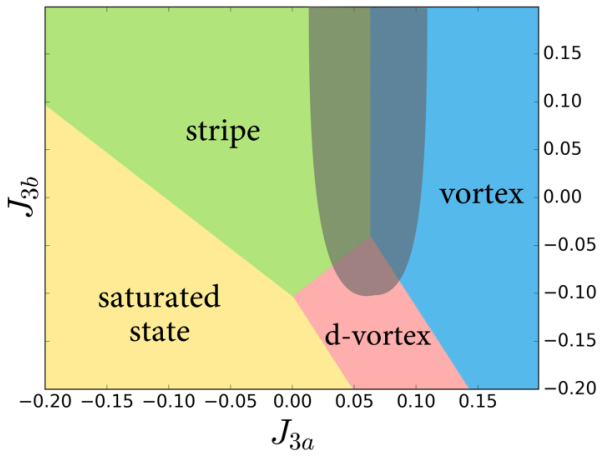

(c) vortex state

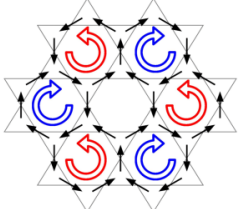

(d) saturated state

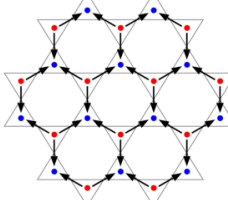

(e) stripe state

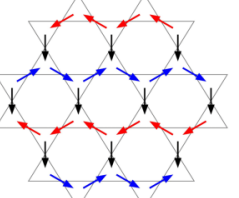

(f) d-vortex state

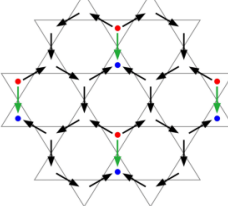

FIG. 2. Energetic phase diagrams and corresponding groundstate configurations. The phase boundaries are calculated based on the energy differences. The first two parameters in the Hamiltonian are fixed as $\left(J_{1}, J_{2}\right)=(1,-1 / 3)$. (a) Different field strengths with fixed $\left(J_{3 a}, J_{3 b}\right)=(0.05,0)$. (b) Different third-neighbor coupling constants with fixed $\left(h_{\perp}, h_{\|}\right)=(11.76,-1.68)$. The snake metastable states occur at the shaded region at finite temperatures. [(c)-(f)] Possible ground states. The green spins in panel (f) denote flipped kink spins in the vortex state in panel (a).

The degeneracy of single-charge configurations is lifted by an out-of-plane magnetic field $h_{\perp}$, which acts as staggered chemical potential of triple charges [Fig. 1(c)]. Since each triangular unit contains two majority spins and one minority spin under ice rule, the configurations with majority spins aligned to the field become energetically more favorable in large perpendicular field. For $h_{\perp}$ larger than the critical value, the minority spin also tends to be aligned with it, leading to a saturated state of staggered triple charges [Fig. 2(d)]. These triple charges correspond to the magnetic monopoles and antimonopoles in the pyrochlore spin ice in the [111] external field, and the saturation of charges represents the second plateau of the two-stage magnetization process described in Sec. I. Thus, the canted moment in our model allows us to imitate the magnetic field responses of the three-dimensional spin ice.

The projected component of canted spins on the kagome plane provides a way to perturb the kagome-ice manifold further through a small in-plane field $h_{\|}$. The direction of this in-plane field is defined to point toward one vertex of the triangle in the kagome lattice, as shown in Fig. 1(a). Thus, for sufficiently high $h_{\|}$, moments on this sublattice tend to align with $h_{\|}$and the other spins are arranged into the local

configurations that preserve the ice rule. We denote these fixed spins as kink spins and the others as stripe spins, as shown by different colors in Fig. 1(b). At temperatures below $J_{1}$, the states satisfying the ice rule are characterized by polarized kink spins pointing downward and rows of parallel stripe spins, either pointing to the left or to the right, which can be described by a local $Z_{2}$ order parameter. This regime is similar to the sliding phase discussed in other frustrated magnets. As $T$ is further lowered, the second-neighbor coupling $J_{2}<0$ breaks the sliding symmetry and gives rise to an antiferromagnetic order of the parallel spin stripes. The resultant twofold symmetric long-range ordered state is called the stripe state, as depicted in Fig. 2(e).

For weaker in-plane fields, the kink spins are no longer pinned and a different long-range ordered state with $\sqrt{3} \times \sqrt{3}$ magnetic unit cell emerges. This so-called vortex state can be seen as an aggregation of hexagonal vortices with opposite chirality, as shown in Fig. 2(c). The same ground state can be found in both the equivalent short-range model with inplane moments [29] and a long-range kagome-ice model with dipolar interactions [27,28].

Figures 2(a) and 2(b) shows the schematic phase diagrams of the ground states found in equilibrium Monte Carlo simulations. Besides the known ground states mentioned above, we found a new ground state emerging within a small region of the parameter space. The configuration of this state is depicted in Fig. 2(f) and can be seen as a vortex state with columnar defects of reversed kink spins; thus we call it the defected vortex state (d-vortex state). Closer inspection finds one-third of the triangles in this configuration contain triplecharge defects, and the others satisfy the kagome-ice rule with staggered single charges. To our knowledge, this is a rare case where the long-range orders of single and triple charges coexist.

Based on the energy difference of these ordered states, we can obtain the critical magnetic fields at zero temperature. For instance, the critical perpendicular field that drives the system from the vortex state into the saturated state is $h_{\perp, c}=12 J_{1}+$ $12 J_{3 a}+6 J_{3 b}$, and the critical parallel field from the vortex state to the stripe state is $h_{\|, c}=3 \sqrt{2} J_{2}-3 \sqrt{2} J_{3 a}$. The $J_{1}$ interaction does not show up in the formula of $h_{\|}$because both ground states satisfy the ice rule. These critical parameters are consistent with the numerical results of equilibrium Monte Carlo simulation in Fig. 3.

In the $J_{1}-J_{2}-h_{\perp}$ model [Figs. 3(a) and 3(b)], we find the critical field $h_{\perp, c}=12 J_{1}$ in both the charge density and the vortex-state order parameter. For $h_{\perp}<6.72$, the total charge density reduces as temperature increases. This indicates that the local excitations are pairs of negative charges. On the contrary, for $h_{\perp}>6.72$ the total charge density increases with temperature since the triple-charge pairs are more favorable. It is worth noting that only in the latter case can we make the connection to the kagome-ice phase in the pyrochlore spin ice since the presence of negative-charge pairs violates the two-in-two-out ice rule.

For the $J_{1}-J_{2}-h_{\perp}-h_{\|}$model [Fig. 3(c)], the critical parallel field $h_{\|, c}=3 \sqrt{2} J_{2}$ calculated from the energy difference of vortex and stripe states is consistent with the stripe-state ordering in the Monte Carlo simulation. With a moderate 

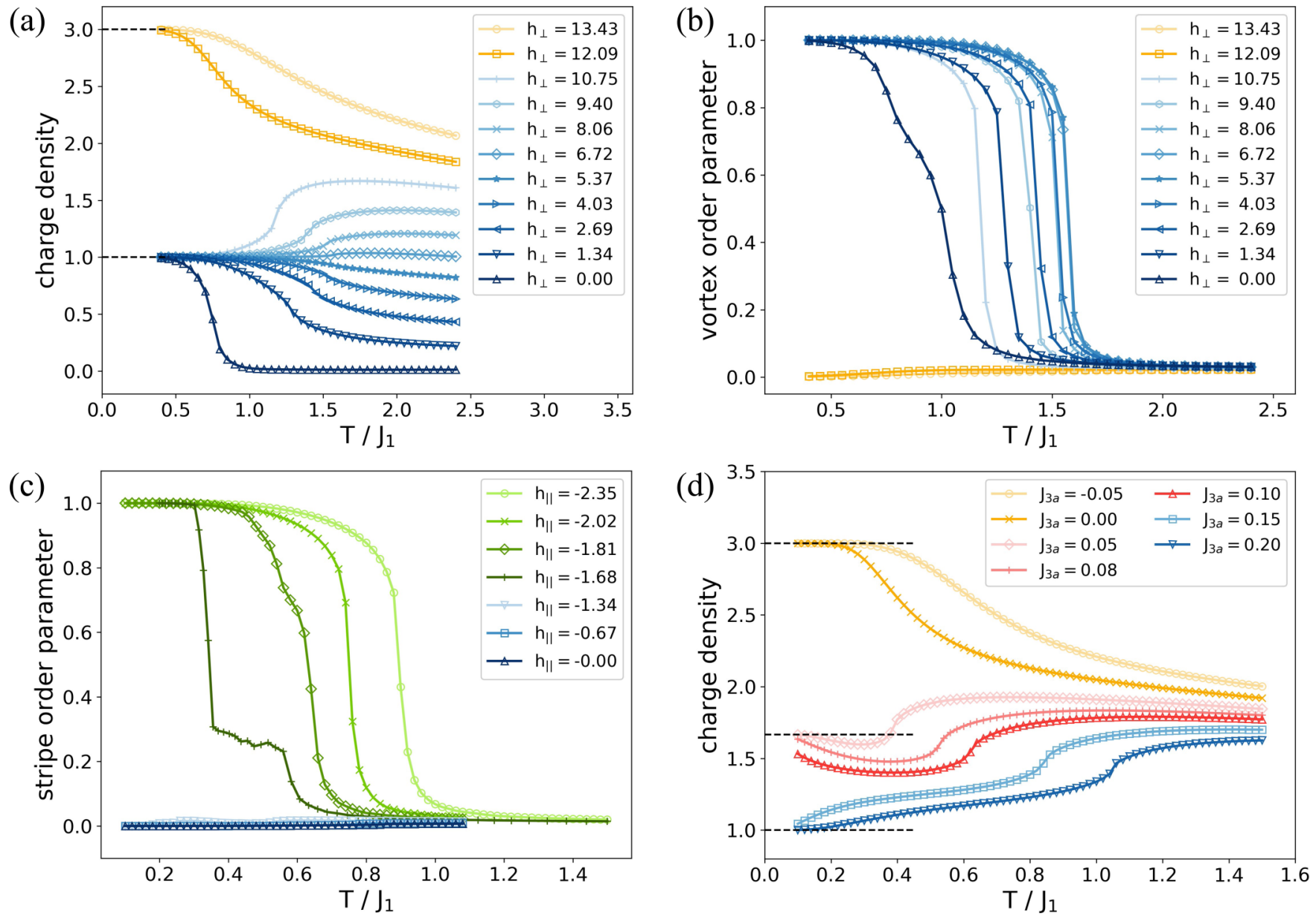

FIG. 3. Equilibrium Monte Carlo results. (a) Charge density and (b) vortex-state order parameter of $J_{1}-J_{2}-h_{\perp}$ model under various $h_{\perp}$. The critical perpendicular field for the ice-rule breaking transition is $h_{\perp}=12 J_{1}=12.00$. (c) Stripe-state order parameter of $J_{1}-J_{2}-h_{\perp}-h_{\|}$model with fixed $h_{\perp}=11.76$ and various $h_{\|}$. The critical parallel field for the stripe state to be the zero-temperature ground state is $h_{\|}=3 \sqrt{2} J_{2}=-1.41$. (d) Charge density of $J_{1}-J_{2}-J_{3}-h_{\perp}-h_{\|}$model with fixed $J_{3 b}=-0.15$ and various $J_{3 a}$. The fields are chosen to be $\left(h_{\perp}, h_{\|}\right)=(11.76,-1.68)$ as in Fig. 2(b).

$h_{\|}$, we observed a mixed phase of vortex and stripe states with numerous metastable states that prevent the system from equilibrating. This metastability plays a central role of this work and will be analyzed in detail in the rest of this paper.

In the following, the parameters of external field are chosen to be $\left(h_{\perp}, h_{\|}\right)=(11.76,-1.68)$. On the one hand, we fix the $h_{\perp}$ to be slightly under $h_{\perp, c}$ to ensure that the ice rule is preserved yet the spins can be easily perturbed since the energy cost of flipping a spin is almost canceled out by the Zeeman energy gain. We use a slightly larger $h_{\|}$than $h_{\|, c}$ in magnitude to ensure that the ground state is the stripe state, which corresponds to $\mathbf{q}=X$ state in the pyrochlore spin ice. Also, these field values imitate the experimental setup of $\mathrm{Ho}_{2} \mathrm{Ti}_{2} \mathrm{O}_{7}$ under tilted [111] fields [24].

\section{FIELD-QUENCH PROCESS}

Since the transitions between various phases in Figs. 2(a) and 2(b) are mostly first order, mixed-phase states with complex structures often emerge at the phase boundaries, especially at finite temperatures. Coupled with the underlying geometrical frustration, metastable states with unusually slow dynamics are observed at the phase boundary between the stripe and the vortex states. Since the relaxation timescale of these metastable states is far beyond the capability of equilibrium Monte Carlo with conventional single-spin-flip or loop algorithms [6], we applied a rejection-free algorithm called the waiting time method (WTM) to investigate the slow dynamics from given initial states [30]. Instead of using the acceptance ratio in the METROPOLIS algorithm, the waiting time method associates to each spin a waiting time as a local indicator of relaxation time, specifying how long a given spin has to wait before flipping. The flipping time for each spin is given by

$$
t_{i}=-\tau_{i} \log X_{i}
$$

where the $X_{i}$ is a random number from the uniform distribution, $X_{i} \in(0,1]$ and $\tau_{i}$ is the waiting time from the Boltzmann weight,

$$
\tau_{i}=\max \left[1, \exp \left(\Delta E_{i} / T\right)\right],
$$

where $\Delta E_{i}$ is the energy cost of flipping the $i$ th spin. Therefore, the local flipping time $t_{i}$ is a stochastic variable from the exponential probability distribution associated with average 
$\tau_{i}$. The global time $t_{g}$ is assigned to the system and updated as $t_{g}=t_{i}$ in each step after the $i$ th spin with lowest $t_{i}$ is flipped. Then, the new waiting time of the flipped spin and its affected neighbors are calculated and added to the local flipping times

$$
t_{i}^{\prime}=t_{g}-\tau_{i}^{\prime} \log X_{i}^{\prime},
$$

where the prime in superscript denotes that the value is generated after flipping the target spin. To present the simulation results, the global time $t_{g}$ is used as the timescale of evolution. Each simulation run is executed at constant temperature and may suffer from metastability at low temperatures. Therefore, averaging measured observables over numerous runs is necessary for obtaining general dynamical behaviors.

By using WTM, we study the $J_{1}-J_{2}-J_{3 a}-h_{\perp}-h_{\|}$model on a $L=72$ kagome lattice with 15552 spins. The field-quench process is built by assigning the initial configuration as a saturated state in the high field $\left(h_{\perp} \gg 12.00\right)$ and then quenching to a lower perpendicular field that prefers the stripe state. The magnitudes of magnetic fields at $t_{g} \geqslant 0$ are chosen to be $\left(h_{\perp}, h_{\|}\right)=(11.76,-1.68)$, along with third-neighbor interaction $J_{3 a}=0.05$ owing to match the energetic phase diagram in Fig. 2(b). We found that it is enough to reach final stage of each simulation by $10^{5}$ Monte Carlo steps, and the results are the average value over 100 samples.

In Fig. 4, we show the time evolution of the system in the field-quench scenario. In addition to the charge density, we measured two ad hoc order parameters to track the evolution of the spin textures. The stripe- and kink-order parameters are defined as

$$
\mathcal{O}_{s}=\left|\sum_{i=1}^{L} v_{i}\left(\sum_{j \in \nearrow, \nwarrow} \eta_{j} \sigma_{j}\right)\right|, \mathcal{O}_{k}=(-1) \sum_{k \in \downarrow} \sigma_{i},
$$

where $\eta= \pm 1$ for different sublattices of stripe spins, and $v=$ \pm 1 for odd and even rows of stripes. The symbols $\nearrow$ and $\nwarrow$ denotes the stripe spins while $\downarrow$ represents kink spins.

As shown in the figure, the relaxation process from the saturated state can be divided into five stages. In stage I, the charge density drops drastically by rapid pair annihilation of triple charges. The stripe order is hardly visible since the emergent domains of single charges are randomly distributed. In the next stage, larger striped domains are constructed by further annihilation and diffusion of triple charges. However, in most simulation runs, the system is stuck in stage III with partial stripe ordering and shows no further evolution. Only in very rare cases can the system in our simulation escape from the long-lived metastable states and reach the stripe ground state in stage $\mathrm{V}$.

\section{THE SNAKE DOMAINS}

The vortex state of the kagome ice is characterized by a broken $Z_{3}$ symmetry on top of the staggered \pm 1 charges. On the other hand, an Ising-like order parameter describes the anisotropic stripe phase. The incompatibility of these two order parameters indicates a first-order phase transition between these two ordered states. As discussed above, complex mixedphase states often emerge at the first-order phase boundary. Here we show that the coexistence regime of the canted kagome ice exhibits an intriguing snake-like structure as well (a)

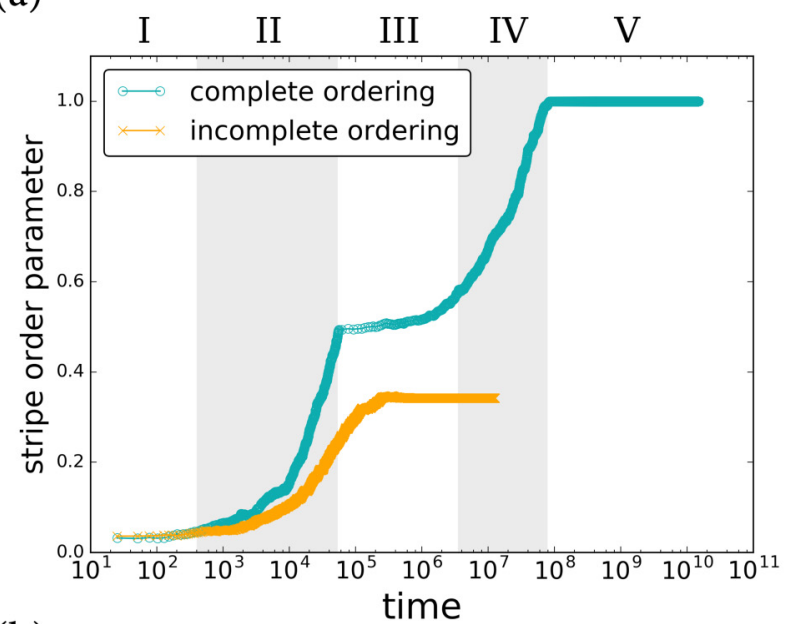

(b)

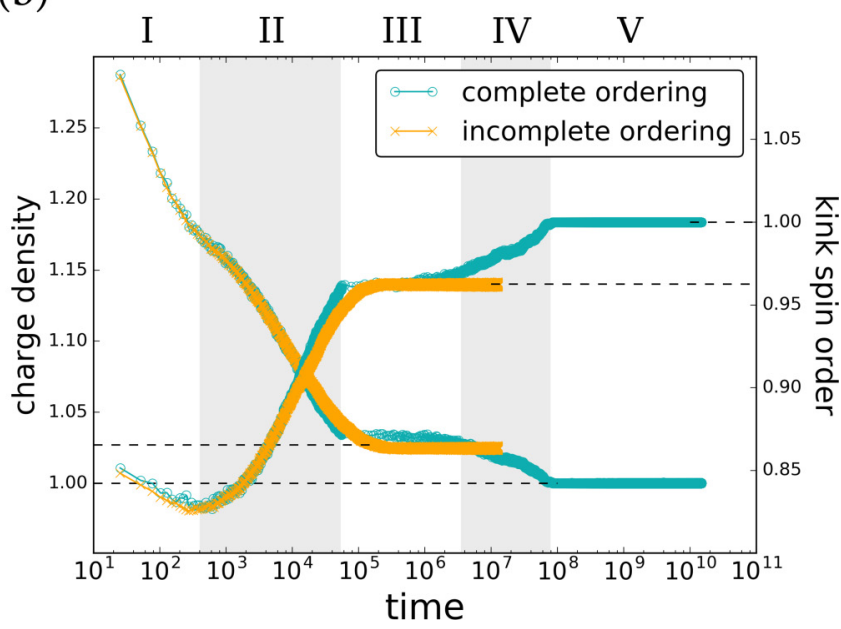

FIG. 4. Field-quench process from the saturated state by waiting time Monte Carlo simulation. (a) Time evolution of stripe order parameter. (b) Time evolution of charge density and kink spin order parameter. The curves of complete ordering show averaged values from those simulations that can reach the stripe state in the end, and the curves of incomplete ordering show averaged values from the systems getting stuck in the snake metastable phase. The timescales are measured from the global time of waiting time method.

as localized "bound" states of monopole-antimonopole pairs at the edges of the snake domains. The appearance of this novel snake structure is related to the emergent gauge-like sliding symmetry of the stripe phase at finite temperatures. A typical structure of a snake domain is illustrated in Fig. 5(b). Because of the presence of further-neighbor interactions, a snake excitation is lower in energy than the string excitation [Fig. 5(a)] and can be seen as a composite of single string and a series of hexagon-loop excitations. Since these hexagonal loops are nonwinding loops and do not change the magnetization, snake and string excitation share the same topological sector and are indistinguishable by their winding numbers $[31,32]$. From a different perspective, the snake domain can be interpreted as two stripe states with opposite chirality, separated by a string-like boundary of vortex state or $d$-vortex state. In this picture, the metastable states of snake domains can be seen as a mixed phase of two competing long-range 
(a)

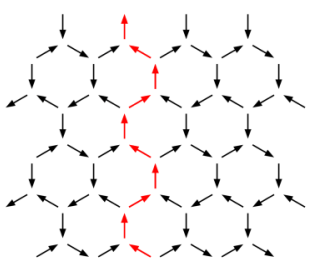

(b)

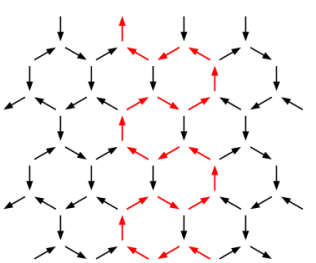

(c)

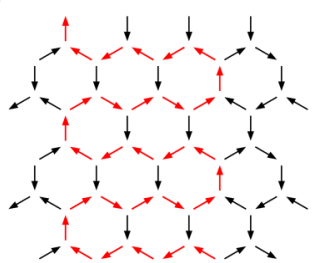

(d)

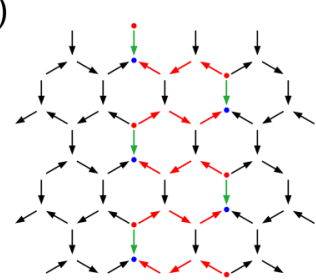

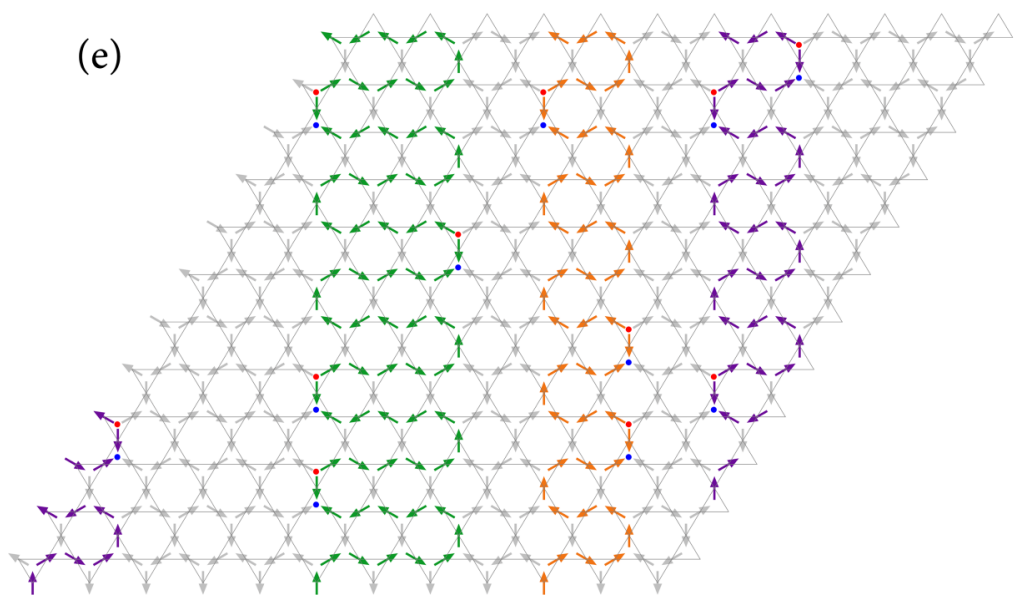

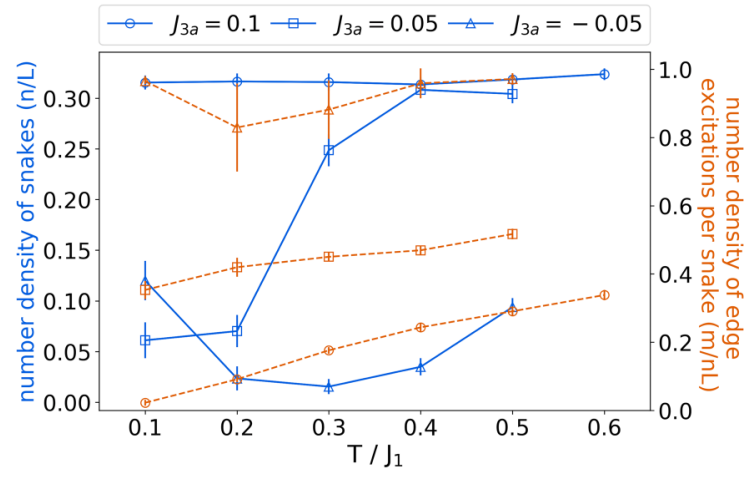

FIG. 5. (a) Single string excitation on the background of the stripe state. (b) Single snake of width $=3$. (c) Single snake of width $=5$. (d) Single defected snake with saturated triple charges on the edge. (e) Representative configuration with multiple snake domains. Red and blue dots denote the thermally driven localized triple charges. Note that the magnetic domains between colored snakes are also snake domains with opposite chirality. (f) Temperature and $J_{3 a}$ dependence of the number density of the snakes $(n / L)$ and the number density of edge excitations per snake $(m / n L)$, calculated by averaging the final values of $n$ and $m$ through Eq. (13) from waiting time Monte Carlo simulations.

ordered states, which explains the emergence of metastability at the periphery of phase boundaries.

Moreover, the width of one snake domain can be any odd number greater than or equal to three, and the snakes with different widths are actually degenerate in energy due to the short-range nature of interactions in our model. The energy change of a single snake from the background stripe state can be written as

$$
\begin{aligned}
E_{\text {snake }} & =E_{\text {stripe }}+\Delta E_{s} L, \\
\Delta E_{s} & =12 J_{2}-12 J_{3 a}-2 \sqrt{2} h_{\|},
\end{aligned}
$$

where $L$ is the lattice dimension along one edge and $\Delta E_{s}$ denotes the energy difference per unit length. Note that for the simulation result presented in Fig. 4, the parameters are chosen to be $\left(J_{2}, J_{3 a}\right)=(-1 / 3,0.05)$ and $h_{\|}=-1.68$. Therefore, the Zeeman energy of in-plane field is the only term that raises the energy from the stripe state. Since the energy increase of forming a snake from the stripe state is proportional to the vertical dimension $L$, it is expected that a perfect snake domain is unlikely to be seen in the thermodynamic limit. However, according to our simulation, a broken snake can only propagate through the migration of single monopoles at the end points. Thus, the kinetic bottleneck of moving one monopole along a highly anisotropic path results in the slow relaxation of these domains even in larger system sizes.

At low temperatures, once a uniform snake domain is formed and wind through the periodic boundaries, spins become very robust against thermal fluctuation except for the kink spins on the edge. When a snake emerges from the stripe state, the ice-rule constraint forces the kink spins on the edge to align opposite to the in-plane field. Flipping those spins via thermal fluctuation can lower the Zeeman energy but simultaneously break the ice rule and create triple-charge pairs. In a large perpendicular field as in the WTM simulations, cancellation of spin-spin interaction and the Zeeman energy allows the edge spins to be more easily thermally flipped, resulting in isolated triple-charge pairs. Interestingly, although the edge spins are easily excited, these charges can hardly diffuse to any adjacent positions. In a sense, these triple charges are not only confined but also localized, partly attributed to the stable structure of the snake domains. The excitation energy of each edge spin can be shown explicitly as

$$
\Delta E_{e}=8 J_{1}+4 J_{2}+4 J_{3 a}+4 J_{3 b}-\frac{2}{3} h_{\perp}-\frac{2 \sqrt{2}}{3} h_{\|}
$$

With sufficiently large $h_{\|}$or $J_{3 b}$, all the edge spins can be excited and aligned with the in-plane field, as shown in Fig. 5(d).

For a metastable state with snake domains, there are two types of disorders. First, both the number of the snake domains and their widths can fluctuate. This contributes to the destruction of the stripe order in our simulation. Second, although thermal fluctuation creates localized edge excitations, the total number of these edge spins is governed by the number of snakes. In general, for a given configuration of $n$ snakes and $m$ edge excitations as shown in Fig. 5(e), the total 

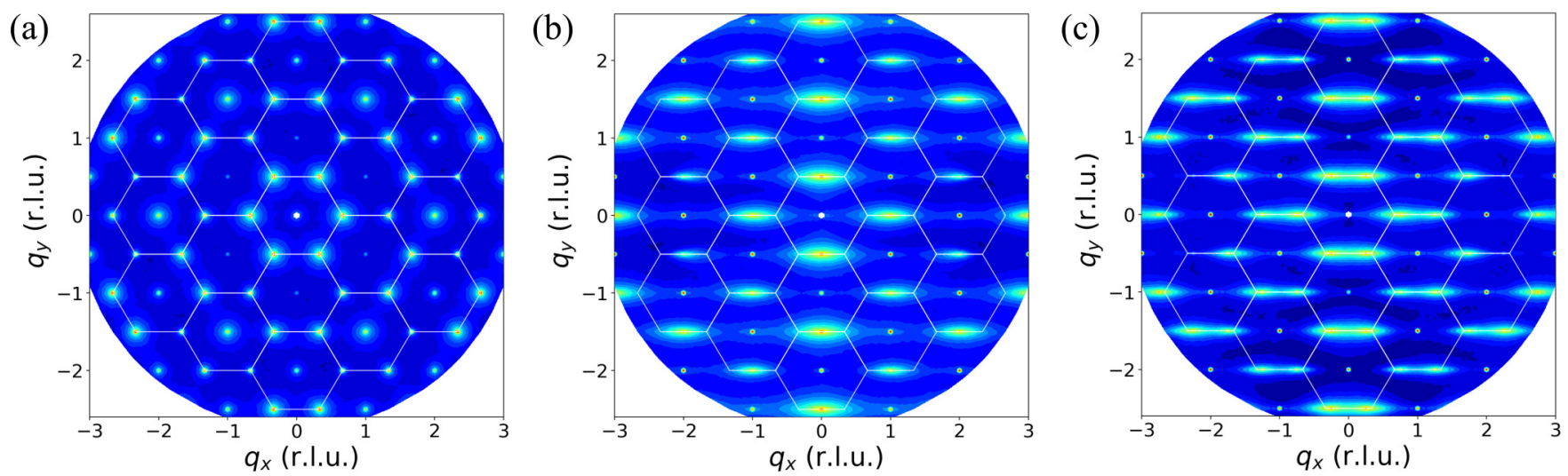

FIG. 6. Magnetic structure factor of the (a) vortex state, (b) stripe state, and (c) mixed phase of snake domains. Note that in panel (c), the highest intensity points on the edge of Brillouin zone locate at the position between the corner and edge center, which are the positions of Bragg peaks of vortex and stripe state, respectively. Metastable states with different superlattice periodicity result in different positions of the peak along the same line.

energy of this configuration is

$$
E_{n, m}=E_{\text {stripe }}+n \Delta E_{s} L+m \Delta E_{e},
$$

where $n$ and $m$ are non-negative integers with the upper bounds $n \leqslant L / 3$ and $m \leqslant n L$. Two distinctive conditions are worth considering. For $n=L / 3$ and $m=0$, all the snake domains have minimum width and all edge spins are not excited. This fully compact configuration of snakes is exactly the vortex state. In a similar way, for $n=L / 3$ and $m=n L$ it is equivalent to a defected vortex state. Therefore, these metastable states can be regarded as mixture of the three ground states. Using the order parameters we measured in the simulations, we can calculate the exact values of $n$ and $m$ via

$$
n=\frac{\left(Q_{t}-\mathcal{O}_{k}\right) L}{2}, m=\frac{\left(Q_{t}-1\right) L^{2}}{2} .
$$

The temperature and $J_{3 a}$ dependence of $n$ and $m$ are presented in Fig. 5(f). We consider the temperature range below $0.6 J_{1}$ because the snake domains are thermally destroyed at higher temperatures and the definitions of $n$ and $m$ are no longer valid. By modeling the surface fluctuation as a square Ising interface without external fields, we find the melting temperature of the snake domains is consistent with the roughening transition temperature (see Appendix B). In almost all the cases, the number of excited edge spins increases monotonically as temperature rises, indicating the isolated and localized nature of these spins. In contrast, the number density of the snakes does not show the same trend and is changed by third-neighbor perturbation. On account of fixed lattice size in our simulations, the number density of snake is inversely proportional to the average size of snake-like domains. For $J_{3 a}=0.1$, the zero-temperature ground state is the vortex state [Fig. 2(b)], and the domain structure is quite robust against thermal fluctuation, showing the saturated value in the number density of snakes (0.33) at all temperatures considered in Fig. 5(f). However, thermal fluctuation does excite the edge spins locally, leading to the monotonic increase in the number of excited edge spins.
On the other hand, for $J_{3 a}=-0.05$, larger snake domains are easier to form since stripe state is the ground state. In this case, we notice that at intermediate temperatures thermal energy can reduce the number density of the snakes and expand the domain width, moving the system closer to the stripe state. Finally, when $J_{3 a}$ is closer to the vortex-stripe phase boundary, the number density of the snakes shows a gradual increase with temperature, indicating that the nucleation process of the snakes is thermally enhanced. Therefore, although the stripe state is the zero-temperature ground state for $J_{3 a}=0.05$, at intermediate temperatures the domain structure is actually closer to the vortex state. At higher temperatures, the domain structure is eventually destroyed by thermal fluctuations.

Finally, we remark on the possible experimental signatures of the snake phase in kagome-ice systems. From the equilibrium Monte Carlo simulations, we calculate the magnetic structure factor for different phases at finite temperatures. On one hand, the Bragg peaks of vortex states appear at the corners of the Brillouin zone, indicating the sixfold symmetric configurations [Fig. 6(a)]. On the other hand, the presence of $h_{\|}$breaks the rotational symmetry and the Bragg peaks only appear at the center of two edges with oval-shaped diffuse scattering [Fig. 6(b)]. Note that this similar pattern is observed in the neutron-scattering experiment of $\mathrm{Ho}_{2} \mathrm{Ti}_{2} \mathrm{O}_{7}$ single crystal under tilted [111] magnetic field [24], and later explained as the presence of long-range ordered $\mathbf{q}=X$ state, which is the three-dimensional analogy of our stripe state [25]. However, the Bragg peaks of $\mathbf{q}=X$ state is not observed in the neutron experiment. For the snake phase in our model, the structure factor shows similar horizontally smeared scattering along the top and bottom edges, but the highest intensity no longer appears at the stripe position due to the presence of snake domains that breaks the periodicity of the stripe spins [Fig. 6(c)]. In one simulation, the points with highest intensity are located at somewhere between the Bragg peaks of two long-range ordered states, indicating the frozen topological sector of kagome-ice manifold.

In the artificial spin ice systems, thermally active kagomeice nanoarray of permalloy is successfully synthesized and studied recently. The equilibrium thermal behaviors such as 
phase transition from kagome-ice phase to long-range ordered vortex state is detected by low-energy muon spin relaxation ( $\mu \mathrm{SR}$ ) [33], while the nonequilibrium field-quench relaxation is also investigated through time-dependent spatial correlation of moments and charges [34]. Most important, through the $\mathrm{x}$-ray photo-emission electron microscopy and magnetic force microscopy, both the magnetic domains and local triplecharge pairs can be imaged in the artificial kagome-ice system $[34,35]$.

\section{CONCLUSION}

In conclusion, we study a kagome-ice model with canted spins that respond to both in-plane and out-of-plane magnetic fields. The inclusion of second- and third-neighbor spin-spin interactions give rise to complex texture in both spin and charge degrees of freedom. The various competing phases at low temperatures lead to novel snake-like structure in the coexistence regime between the vortex and stripe phases. The combination of geometrical frustration and the anisotropic nature of these snake domains give rise to robust metastable states with extremely slow relaxation dynamics after the field quench. We provide detailed structural and energetic characterizations of these snake domains in the mixed-phase states. In spite of its long-lived nature, spins on the edge of each domain can be easily excited by thermal fluctuation, leading to frequent creation and annihilation of triple charges. These topological charges are localized since they can hardly overcome the energy barrier to migrate into the interior of the snake domain.

The structure of the mixed-phase states also plays an important role in the kinetics of the first-order phase transitions. For example, in the nucleation and growth scenario, the surface tension of domain walls enclosing the stable phase determines the size distribution of the nuclei as well as the nucleation speed. The elongated structure of the snake domains implies a rather anisotropic nucleation process as well as relaxation dynamics, which should be rather different from the isotropic case and is an interesting subject for future studies.

Similar metastability with more complicated spin textures was reported in an extensive Monte Carlo study of the dipolar kagome ice phase on a pyrochlore lattice [36]. Our canted kagome ice model serves as a minimum model to demonstrate the emergent metastability of spin ice under tilted [111] fields. From the experimental perspective, these metastabilities and domain structures may be relevant to the absence of $\mathbf{q}=X$ Bragg peak in neutron scattering experiments [24]. Also, the recently discovered kagome-layered compound $\mathrm{Dy}_{3} \mathrm{Mg}_{2} \mathrm{Sb}_{3} \mathrm{O}_{14}$ [14] confirms the charge-ordering transition of kagome ice and can potentially demonstrate the metastability and charge texture when external field is applied.

\section{ACKNOWLEDGMENTS}

This work was supported in part by the Ministry of Science and Technology (MOST) of Taiwan under Grants No. 105-2112-M-002-023-MY3, No. 107-2112-M-002 -016 MY3 (W.H.K., Y.J.K.), and No. 108-2918-I-002 -032 (Y.J.K.).
This work was performed in part at Aspen Center for Physics, which is supported by National Science Foundation Grant No. PHY-1607611, and was partially supported by a grant from the Simons Foundation. We are also grateful to the National Center for High-Performance Computing in Taiwan for computer time and facilities.

\section{APPENDIX A: KAGOME ICE MODEL DERIVED FROM PYROCHLORE SPIN ICE MODEL}

Here we demonstrate the two-dimensional $J_{1}-J_{2}$ kagome ice model derived from the pyrochlore spin ice when spins on one of the four sublattice sites of each tetrahedron is pinned. We start from a pyrochlore spin ice with effective $J_{1}$ and $J_{2}$ interactions. Physically each effective coupling strength comes from the sum of dipolar interaction and the exchange interaction. We begin with the $J_{1}-J_{2}$ Hamiltonian on a pyrochlore lattice:

$$
\mathcal{H}=\mathcal{H}_{1}+\mathcal{H}_{2}=J_{1} \sum_{\langle i j\rangle} \sigma_{i} \sigma_{j}+J_{2} \sum_{\langle\langle i j\rangle\rangle} \sigma_{i} \sigma_{j},
$$

where $\sigma_{i}=+1(-1)$ denotes a Ising spin pointing out of (into) a tetrahedron. Note that each spin is shared by two neighboring tetrahedra; thus, a spin pointing into one implies pointing out of the other. Therefore, we use the convention that the pyrochlore lattice is decomposed into two kinds of tetrahedra, and the $\sigma_{i}$ is defined with respect to one kind of them.

Next, we separate the summation of sites in $\mathcal{H}_{1}$ into summations of different sublattice indices. We denote $T$ as a set of spins located on the triangular planes and $K$ for the sites located on the kagome planes:

$$
\begin{aligned}
\mathcal{H}_{1} & =\frac{J_{1}}{2} \sum_{i} \sigma_{i}\left(\sum_{j \in \mathrm{NN}(i)} \sigma_{j}\right) \\
& =\frac{J_{1}}{2}\left\{\sum_{i \in \mathrm{T}} \sum_{j \in \mathrm{NN}(i)} \sigma_{j}+\sum_{i \in \mathrm{K}} \sigma_{i}\left(\sum_{\substack{j \in \mathrm{NN}(i) \\
j \in \mathrm{K}}} \sigma_{j}+2\right)\right\} \\
& =\frac{J_{1}}{2}\left\{2 \sum_{i \in \mathrm{K}} \sigma_{i}+\sum_{i \in \mathrm{K}} \sigma_{i}\left(\sum_{\substack{j \in \mathrm{NN}(i) \\
j \in \mathrm{K}}} \sigma_{j}\right)+2 \sum_{i \in \mathrm{K}} \sigma_{i}\right\} \\
= & J_{1} \sum_{\substack{\langle i, j\rangle \\
i, j \in \mathrm{K}}} \sigma_{i} \sigma_{j}+2 J_{1} \sum_{i \in \mathrm{K}} \sigma_{i} .
\end{aligned}
$$

On the second line, we exploit the fact that all spins on triangular planes are pinned in the kagome ice system, such that $\sigma_{i \in T}=+1$. Therefore, the Hamiltonian is reduced to a two-dimensional model with nearest-neighbor coupling and an effective Zeeman term. 
For the second term $\mathcal{H}_{2}$, the same decomposition can be applied:

$$
\begin{aligned}
\mathcal{H}_{2}= & \frac{J_{2}}{2} \sum_{i} \sigma_{i}\left(\sum_{j \in \mathrm{NNN}(i)} \sigma_{j}\right) \\
= & \frac{J_{2}}{2}\left\{\sum_{i \in \mathrm{T}}\left(\sum_{\substack{j \in \mathrm{NNN}(i) \\
j \in \mathrm{K}}} \sigma_{j}\right)+\sum_{i \in \mathrm{K}} 4 \sigma_{i}\right. \\
& \left.+\sum_{i \in \mathrm{K}} \sigma_{i}\left(\sum_{\substack{j \in \mathrm{NNN}(i) \\
j \in \mathrm{K} \\
\text { intraplane }}} \sigma_{j}\right)+\sum_{i \in \mathrm{K}} \sigma_{i}\left(\sum_{\substack{j \in \mathrm{NNN}(i) \\
j \in \mathrm{K} \\
\text { interplane }}} \sigma_{j}\right)\right\} \\
= & J_{2} \sum_{\substack{\langle\langle i, j\rangle\rangle \\
i, j \in \mathrm{K}}} \sigma_{i} \sigma_{j}+4 J_{2} \sum_{i \in \mathrm{K}} \sigma_{i}+J_{2} \sum_{\substack{\langle\langle i, j\rangle\rangle \\
i, j \in \mathrm{K} \\
\text { interplane }}} \sigma_{i} \sigma_{j} .
\end{aligned}
$$

The last term shows the second-neighbor interactions between different kagome planes. Since we consider only one kagome plane in this work, these interplane bonds are not taken into account in our model. Therefore, we obtain the reduced $J_{1}-J_{2}$ Hamiltonian on one kagome plane:

$$
\begin{aligned}
\mathcal{H}= & J_{1}\left(\sum_{\substack{\langle i, j\rangle \\
i, j \in \mathrm{K}}} \sigma_{i} \sigma_{j}\right)+J_{2}\left(\sum_{\substack{\langle\langle i, j\rangle\rangle \\
i, j \in \mathrm{K}}} \sigma_{i} \sigma_{j}\right) \\
& +\left(2 J_{1}+4 J_{2}\right) \sum_{i \in \mathrm{K}} \sigma_{i} .
\end{aligned}
$$

Note that when $J_{1}$ and $J_{2}$ are opposite in sign, the Zeeman coupling strength is reduced. In this work, we choose $J_{1}=1$ and $J_{2}=-1 / 3$, and the overall positive coupling constant makes the saturated state more favorable when considering only the Zeeman term. Therefore, the consequence of the effective Zeeman term is lowering the critical external field of ice-rule breaking transition.

Similar derivation can be made for the third-neighbor interactions. However, the third-neighber interactions on the pyrochlore only couple the spins on the same sublattice sites. As a result, there is no effective Zeeman term derived from the third-neighbor bonds. Again, in the effective two-dimensional model we include the intraplane third-neighbor bonds but ignore the interplane bonds.

\section{APPENDIX B: ROUGHENING OF SNAKE DOMAINS}

For snake domains without any excited edge spins, the whole configuration satisfies the kagome-ice rule on each triangular plaquette. Under this circumstance, the domain walls can fluctuate through the loop-like flips without breaking the ice rule, as depicted in Fig. 7(a). For one hexagonal excitation out of smooth domain walls, the energy increases by

$$
\Delta E_{\mathrm{hex}}=-8 J_{2}+8 J_{3 a} .
$$

(a)

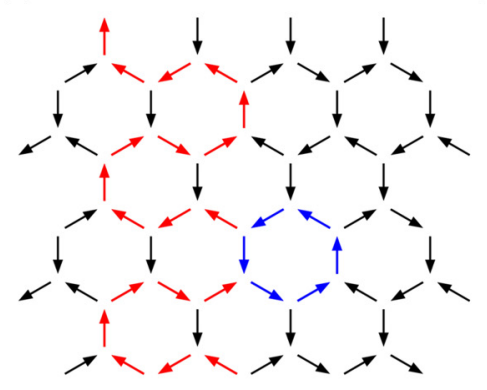

(b)

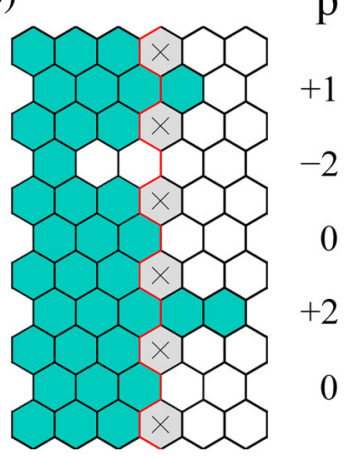

(c)

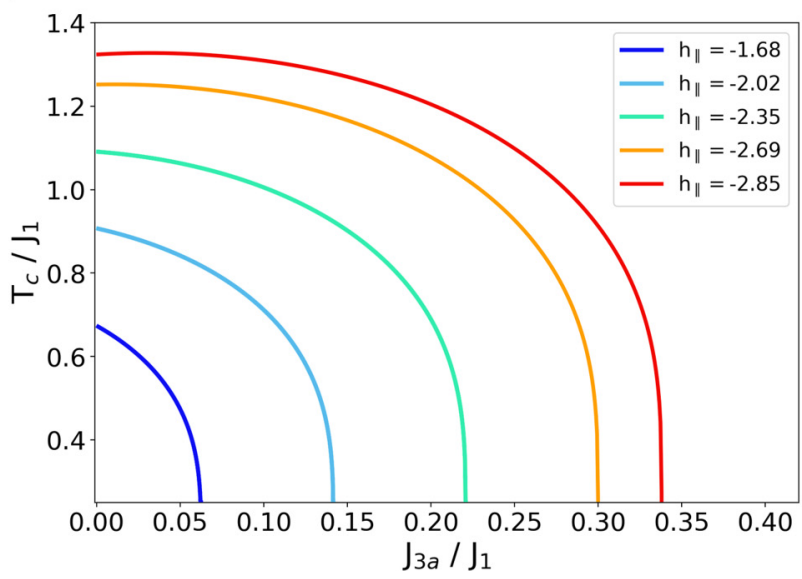

FIG. 7. The snake domain roughening. (a) On a given smooth wall of snake domains, the hexagon loop can locally change the width of domains without breaking the ice rule. (b) Rough interface. The red line denotes the smooth interface with zero height $(p=0)$. Only the hexagon loops on the even rows can be excited, so that the difference in height is calculated between adjacent even rows. (c) Roughening transition temperature calculated from MüllerHartmann-Zittartz method. Curves from left to right present the results from lower fields to higher fields.

Note that this hexagonal excitation does not change the total moment along both $h_{\perp}$ and $h_{\|}$such that $\Delta E_{\text {hex }}$ depends only on the further-neighbor couplings. For a given system with one string-like and smooth interface that separates the two snake domains with different chirality, the internal energy is $E_{0}+\Delta E_{s} L / 2$, where $E_{0}$ is the ground-state energy of stripe state and $\Delta E_{s}$ is the energy increment per unit length of a snake defined in Sec. V. For each even row of the interface, the hexagonal excitations can move the domain wall either to the left or to the right, as shown in Fig. 7(b). Thus, we define the height of each even row as the number of hexagonal excitations $p$ with respect to the original smooth interface. Therefore, for a rough interface, the total energy increment depends only on the difference of height between adjacent even rows:

$$
\Delta E_{r}=\sum_{i=1} \frac{1}{2} \Delta E_{\mathrm{hex}}\left|p_{2 i}-p_{2 i+2}\right|
$$

with the partition function $Z=\sum_{\left\{p_{i}\right\}} e^{-\beta \Delta E_{r}\left\{p_{i}\right\}}$. This surface fluctuation can be mapped into a square Ising interface 
without external fields. Here we apply the Müller-HartmannZittartz method [37] to estimate the roughening transition temperature from the interfacial free energy:

$$
\begin{aligned}
\sigma & =\frac{1}{4} \Delta E_{s}-T \lim _{L \rightarrow \infty} \frac{1}{L} \ln Z \\
& =\frac{1}{4} \Delta E_{s}-T \ln \lambda_{\max },
\end{aligned}
$$

where $\lambda_{\max }$ is the largest eigenvalue of the transfer matrix $\mathcal{T}$ with matrix elements $\mathcal{T}_{a b}=\exp \left(-\beta \Delta E_{\text {hex }} / 2\right)$ and $a, b=$ $\{0, \pm 1, \pm 2, \ldots, \pm \infty\}$. Another factor of $1 / 2$ is added to the first term of $\sigma$ because hexagonal excitations can only take place on even rows. Finally, we obtain the transcendental equation of the transition temperature $T_{c}$ from $\sigma=0$ :

$$
\frac{6 J_{2}-6 J_{3 a}-\sqrt{2} h_{\|}}{2 T_{c}}=\ln \left[\operatorname{coth}\left(\frac{\Delta E_{\mathrm{hex}}}{2 T_{c}}\right)\right] \text {. }
$$

The $h_{\|}$and $J_{3 a}$ dependence of roughening transition $T_{c}$ is shown in Fig. 7(c). The Zeeman energy from $h_{\|}$contributes to the internal energy cost of a smooth interface, and larger $h_{\|}$in magnitude in fact lowers the roughening temperature since it stabilizes the stripe state. In contrast, $J_{3 a}$ contributes to both the creation of smooth interface and the interfacial fluctuations, so smooth domain walls with more interfaces are favored. Therefore, larger $J_{3 a}$ leads to the vortex state and reduces the roughening temperature. This roughening transition temperature gives us the temperature scale of snakedomain formation and is consistent with our Monte Carlo results.
[1] S. F. Edwards and P. W. Anderson, J. Phys. F: Met. Phys. 5, 965 (1975).

[2] M. J. Harris, S. T. Bramwell, D. F. McMorrow, T. Zeiske, and K. W. Godfrey, Phys. Rev. Lett. 79, 2554 (1997).

[3] A. P. Ramirez, A. Hayashi, R. J. Cava, R. Siddharthan, and B. S. Shastry, Nature (London) 399, 333 (1999).

[4] S. V. Isakov, K. Gregor, R. Moessner, and S. L. Sondhi, Phys. Rev. Lett. 93, 167204 (2004).

[5] S. V. Isakov, R. Moessner, and S. L. Sondhi, Phys. Rev. Lett. 95, 217201 (2005).

[6] R. G. Melko, B. C. den Hertog, and M. J. P. Gingras, Phys. Rev. Lett. 87, 067203 (2001).

[7] C. Castelnovo, R. Moessner, and S. L. Sondhi, Nature (London) 451, 42 (2008).

[8] D. J. P. Morris, D. A. Tennant, S. A. Grigera, B. Klemke, C. Castelnovo, R. Moessner, C. Czternasty, M. Meissner, K. C. Rule, J.-U. Hoffmann, K. Kiefer, S. Gerischer, D. Slobinsky, and R. S. Perry, Science 326, 411 (2009).

[9] C. L. Henley, Annu. Rev. Condens. Matter Phys. 1, 179 (2010).

[10] T. Fennell, P. P. Deen, A. R. Wildes, K. Schmalzl, D. Prabhakaran, A. T. Boothroyd, R. J. Aldus, D. F. McMorrow, and S. T. Bramwell, Science 326, 415 (2009).

[11] M. E. Brooks-Bartlett, S. T. Banks, L. D. C. Jaubert, A. Harman-Clarke, and P. C. W. Holdsworth, Phys. Rev. X 4, 011007 (2014).

[12] S. Petit, E. Lhotel, B. Canals, M. Ciomaga Hatnean, J. Ollivier, H. Mutka, E. Ressouche, A. R. Wildes, M. R. Lees, and G. Balakrishnan, Nat. Phys. 12, 746 (2016).

[13] Z. L. Dun, J. Trinh, K. Li, M. Lee, K. W. Chen, R. Baumbach, Y. F. Hu, Y. X. Wang, E. S. Choi, B. S. Shastry, A. P. Ramirez, and H. D. Zhou, Phys. Rev. Lett. 116, 157201 (2016).

[14] J. A. Paddison, H. S. Ong, J. O. Hamp, P. Mukherjee, X. Bai, M. G. Tucker, N. P. Butch, C. Castelnovo, M. Mourigal, and S. E. Dutton, Nat. Commun. 7, 13842 (2016).

[15] B. Canals, I.-A. Chioar, V.-D. Nguyen, M. Hehn, D. Lacour, F. Montaigne, A. Locatelli, T. O. Menteş, B. S. Burgos, and N. Rougemaille, Nat. Commun. 7, 11446 (2016).

[16] M. Udagawa, L. D. C. Jaubert, C. Castelnovo, and R. Moessner, Phys. Rev. B 94, 104416 (2016).
[17] T. Mizoguchi, L. D. C. Jaubert, and M. Udagawa, Phys. Rev. Lett. 119, 077207 (2017).

[18] I. A. Chioar, N. Rougemaille, and B. Canals, Phys. Rev. B 93 , 214410 (2016).

[19] A. Smerald, S. Korshunov, and F. Mila, Phys. Rev. Lett. 116, 197201 (2016).

[20] P. Mellado, O. Petrova, Y. Shen, and O. Tchernyshyov, Phys. Rev. Lett. 105, 187206 (2010).

[21] I. A. Chioar, B. Canals, D. Lacour, M. Hehn, B. Santos Burgos, T. O. Menteş, A. Locatelli, F. Montaigne, and N. Rougemaille, Phys. Rev. B 90, 220407(R) (2014).

[22] F. Montaigne, D. Lacour, I. A. Chioar, N. Rougemaille, D. Louis, S. M. Murtry, H. Riahi, B. S. Burgos, T. O. Menteş, A. Locatelli, B. Canals, and M. Hehn, Sci. Rep. 4, 5702 (2014).

[23] K. Matsuhira, Z. Hiroi, T. Tayama, S. Takagi, and T. Sakakibara, J. Phys.: Condens. Matter 14, L559 (2002).

[24] T. Fennell, S. Bramwell, D. McMorrow, P. Manuel, and A. Wildes, Nat. Phys. 3, 566 (2007).

[25] W.-H. Kao, P. C. W. Holdsworth, and Y.-J. Kao, Phys. Rev. B 93, 180410(R) (2016).

[26] In order to compare the canted kagome-ice model with pyrochlore spin ice, it is worth noting that $h_{\perp}$ and $h_{\|}$refer to the field strengths along the [111] and [ $\overline{1} \overline{1} 2]$ crystallographic directions of pyrochlore, respectively. Therefore, the negative value of $h_{\|}$in our model corresponds to the experimentally interesting negative tilt of [111] field toward [112]] direction.

[27] G.-W. Chern, P. Mellado, and O. Tchernyshyov, Phys. Rev. Lett. 106, 207202 (2011).

[28] G.-W. Chern and O. Tchernyshyov, Philos. Trans. R. Soc., A 370, 5718 (2012).

[29] A. S. Wills, R. Ballou, and C. Lacroix, Phys. Rev. B 66, 144407 (2002).

[30] J. Dall and P. Sibani, Comput. Phys. Commun. 141, 260 (2001).

[31] A. J. Macdonald, P. C. Holdsworth, and R. G. Melko, J. Phys.: Condens. Matter 23, 164208 (2011).

[32] L. D. C. Jaubert, M. J. Harris, T. Fennell, R. G. Melko, S. T. Bramwell, and P. C. W. Holdsworth, Phys. Rev. X 3, 011014 (2013). 
[33] L. Anghinolfi, H. Luetkens, J. Perron, M. G. Flokstra, O. Sendetskyi, A. Suter, T. Prokscha, P. M. Derlet, S. L. Lee, and L. J. Heyderman, Nat. Commun. 6, 8278 (2015).

[34] A. Farhan, P. M. Derlet, L. Anghinolfi, A. Kleibert, and L. J. Heyderman, Phys. Rev. B 96, 064409 (2017).
[35] K. Zeissler, M. Chadha, E. Lovell, L. F. Cohen, and W. R. Branford, Sci. Rep. 6, 30218 (2016).

[36] B. Yee, Magnetic ordering of dipolar spin ice in moderate [111] field, Master's thesis, University of Waterloo, Waterloo, Ontario, Canada, 2016.

[37] E. Müller-Hartmann and J. Zittartz, Z. Phys. B 27, 261 (1977). 